\title{
Dinâmica populacional de fungos causadores de doenças de tronco em videiras sob a ação de possíveis agentes inibidores
}

\section{Population dynamics of fungi that cause stem diseases in vines under the action of possible inhibitory agents}

\begin{abstract}
Lisandra Pitol Instituto Federal de Educação, Ciência e Tecnologia do Rio Grande do Sul (IFRS), Campus Bento Gonçalves, Bento Gonçalves, RS, Brasil https://orcid.org/0000-0003-2717-031X, lisandrapitol@gmail.com
\end{abstract}

Tatiane Weimann Instituto Federal de Educação, Ciência e Tecnologia do Rio Grande do Sul (IFRS), Campus Bento Gonçalves, Bento Gonçalves, RS, Brasil https://orcid.org/0000-0003-0505-9805, tatiane.weimann@gmail.com

Sandra Denise Stroschein Instituto Federal de Educação, Ciência e Tecnologia do Rio Grande do Sul (IFRS), Campus Bento Gonçalves, Bento Gonçalves, RS, Brasil https://orcid.org/0000-0003-3292-2491, sandra.stroschein@bento.ifrs.edu.br

Marcus André Kurtz Almança Instituto Federal de Educação, Ciência e Tecnologia do Rio Grande do Sul (IFRS), Campus Bento

Gonçalves, Bento Gonçalves, RS, Brasil https://orcid.org/0000-0002-3895-4390, marcus.almanca@bento.ifrs.edu.br Cristian Schweitzer de Oliveira
Instituto Federal de Educação, Ciência e Tecnologia do Rio Grande do Sul (IFRS), Campus
Farroupilha, Farroupilha, RS, Brasil
(D) https://orcid.org/0000-0001-8688-3040, cristian.oliveira@farroupilha.ffrs.edu.br

Rodrigo Stiehl Instituto Federal de Educação, Ciência e Tecnologia do Rio Grande do Sul (IFRS), Campus Bento Gonçalves, Bento Gonçalves, RS, Brasil https://orcid.org/0000-0002-8988-2710, rstiehl@outlook.com

\section{Informações do Artigo}

Como citar este artigo

PITOL, Lisandra; WEIMANN, Tatiane; STROSCHEIN, Sandra Denise; ALMANÇA, Marcus André Kurtz; OLIVEIRA, Cristian Schweitzer de; STIEHL, Rodrigo. Dinâmica populacional de fungoscausadores de doenças de tronco em videiras sob a ação de possíveis agentes inibidores. REMAT: Revista Eletrônica da Matemática, Bento Gonçalves, RS, v. 7, n. 1, p. e3003, 12 jan. 2021. DOI:

https://doi.org/10.35819/remat2021v7i1id4432

Histórico do Artigo

Submissão: 18 de agosto de 2020.

Aceite: 9 de novembro de 2020 .

\begin{abstract}
Resumo
O artigo apresenta contribuições matemáticas para as áreas da agricultura e da fitopatologia, utilizando-se de dados reais e modelando o comportamento de fenômenos físicos. O objetivo do trabalho é modelar o crescimento do Botryosphaeria dothidea em meio de cultura com diferentes componentes, comparando os dados experimentais com o modelo matemático clássico de Verhulst. $O$ experimento foi realizado no Laboratório de Fitopatologia do IFRS - Campus Bento Gonçalves, onde foi preparado o meio de cultura batata-dextrose-ágar (BDA), com diferentes componentes possivelmente inibitórios de crescimento, além de uma testemunha (somente com BDA). O meio de cultura foi alocado em placas de Petri e, em seguida, o fungo Botryosphaeria dothidea foi repicado no centro das placas sobre esse meio. Foram realizadas medições do crescimento fúngico a cada 12 horas. Para o processamento numérico e para a exibição dos gráficos foram utilizados softwares computacionais, tais como o Excel e o Scilab. Por meio de Modelos Matemáticos, que
\end{abstract}




\section{Palavras-chave}

Modelo de Verhulst

Fungos

Equações Diferenciais

Modelagem Matemática

Matemática Aplicada consequentemente refletem em Equações Diferenciais Ordinárias, foi possível programar as simulações numéricas, verificando-se, assim, que a testemunha foi a que apresentou menor erro em relação ao modelo clássico. É plausível afirmar que o crescimento do fungo segue um modelo matemático clássico (Verhulst). Porém, foi possível constatar que os meios de cultura que possuíam possíveis inibidores em sua composição retardaram o crescimento do fungo, indicando que neste tipo de situação o modelo matemático clássico não descreve precisamente a realidade, necessitando ser adaptado.

\section{Keywords}

Verhulst' Model

Fungus

Differential Equations

Mathematical Modeling

Applied Mathematics

\begin{abstract}
The article introduces mathematical contributions for the areas of agriculture and phytopathology using actual data and molding the behavior of physical phenomena. The purpose of the work was molding the growth of Botryosphaeria dothidea in culture medium with different components, comparing the experimental data with the classical mathematical model of Verhulst. The experiment was made in the Phytopathology Laboratory - IFRS/Campus Bento Gonçalves, where the culture medium Potato Dextrose Agar (PDA) was prepared with different components that possibly inhibiting growth, besides evidence (exclusively with PDA). The culture medium was allocated in Petri plates and next the fungus Botryosphaeria dothidea was cut up in the center of the plates about this medium. Fungal growth measurements were executed every 12 hours. For the numerical processing and display graphics were used computer software, such as Excel and Scilab. Through Mathematical Models, that consequently reflect in Ordinary Differential Equations, numerical simulations were possible to program, so verifying that the witness showed the smallest mistake in relation to the classical model. It is possible to declare that the fungus growth follows a classical mathematical model (Verhulst). However, it was possible to verify that the culture media that presented possible inhibitors in their composition delayed the growth of the fungus, showing that in this type of situation the classic mathematical model does not describe reality accurately, needing to be adapted.
\end{abstract}

\section{Introdução}

O cultivo de videiras para a produção vitivinícola é algo bastante recorrente na região da Serra Gaúcha. É desse cultivo que muitos produtores rurais ligados à agricultura familiar dependem para sobreviver. Dessa forma, são vários os fatores que podem interferir tanto na qualidade, quanto na quantidade da safra, tais como fatores climáticos e a ocorrência de doenças, como é o caso das doenças fúngicas (ALMANÇA; LERIN; CAVALCANTI, 2015).

Com o intuito de conhecer o comportamento de fungos causadores de doenças de tronco em videiras, faz-se necessário aprofundar os estudos sobre a cinética de crescimento utilizando uma espécie relacionada a estas doenças, em específico o fungo Botryosphaeria dothidea, causador da doença conhecida por podridão descendente.

Em fitopatologia utilizam-se meios de cultura artificiais para cultivo dos fungos em laboratório. O meio de cultura contém nutrientes e vitaminas indispensáveis para o crescimento e 
a reprodução de microrganismos, neste caso, os fungos. Entre os diversos tipos, optou-se por utilizar como base o meio batata-dextrose-ágar (BDA), pelo fato de ser um

meio universal, pois suporta o crescimento da maioria dos fungos, sendo, por isso, usado mundialmente como meio de rotina em laboratórios de Fitopatologia, principalmente para isolamento e manutenção temporária de culturas. (ALFENAS; MAFIA, 2016, p. 39).

A fim de mensurar o comportamento de crescimento fúngico em diferentes condições, o uso da modelagem matemática é uma possibilidade. De acordo com Bassanezi (2009), a modelagem matemática é utilizada com a finalidade de obter e autenticar modelos matemáticos. Refere-se, portanto, à obtenção de modelos matemáticos que generalizam situações reais, buscando descrever mais fielmente possível a realidade estudada.

Dessa forma, utilizando-se da modelagem matemática será possível a comparação dos dados experimentais com um modelo matemático clássico. Também será plausível fazer análises mais detalhadas, possibilitando a realização de ensaios experimentais com foco na busca de alternativas de controle e manejo destas doenças.

A composição do meio de cultura pode alterar a cinética de crescimento e, consequentemente, o modelo matemático que explica esta cinética. Perin et al. (2019) observaram que isolados de Neofusiccocum parvum e Botryosphaeria dothidea, crescendo em meio de cultura BDA, seguem o modelo matemático proposto por Verhulst. No entanto, a modificação dos componentes do meio de cultura, neste caso, possíveis agentes inibidores de crescimento, podem alterar a cinética e o modelo matemático envolvido. Dessa forma, para a obtenção de um modelo mais próximo da realidade, seriam necessárias adaptações, com a incrementação de novos fatores ao modelo pré-estabelecido. No entanto, esses fatores são bastante intrincados, podendo ser relacionados tanto ao meio de cultura como a fatores abióticos. Segundo Bassanezi (2009), a maior dificuldade em modelar fenômenos biológicos se deve ao fato de que esses fenômenos se comportam, muitas vezes, de forma bastante aleatória.

Pelo fato de as publicações nesse sentido serem escassas, tanto relacionadas ao uso de possíveis agentes inibidores de crescimento, quanto uso do modelo matemático para descrever o comportamento do fungo, o objetivo do trabalho é modelar o crescimento do Botryosphaeria dothidea em meio de cultura com diferentes componentes, comparando os dados experimentais com o modelo matemático de Verhulst.

\section{Referencial teórico}

O fenômeno do crescimento fúngico está estritamente ligado à área da Biologia, que por vezes, aos olhos de um leigo, parece se distanciar bastante da Matemática. Conforme Evelyn C. Pielou (1977 apud ZILL; CULLEN, 2001, p. 135) "o fato de que a ecologia é essencialmente um assunto matemático é cada vez mais aceito". Dessa forma, o presente trabalho encontra-se inserido no contexto da Biomatemática, também conhecida como Ecologia Matemática, que nada 
mais é do que, de forma bastante simplificada, as tentativas de representação matemática de fenômenos biológicos. Segundo Zill e Cullen (2001, p. 135),

Historicamente, o primeiro e talvez mais importante ramo da ecologia matemática é a investigação da dinâmica populacional: como populações crescem e decrescem. Equações diferenciais de primeira ordem têm sido uma ferramenta crucial nesse estudo.

Por se tratar de um estudo em que a população aumenta a cada período de tempo, foi necessário o estudo de modelos matemáticos relacionados à dinâmica populacional. Assim, "uma equação diferencial que descreve algum processo físico é chamada, muitas vezes, de modelo matemático do processo [...]" (BOYCE; DIPRIMA, 2006, p. 01). De acordo com Bassanezi (2009), "a modelagem matemática consiste na arte de transformar problemas da realidade em problemas matemáticos e resolvê-los interpretando suas soluções na linguagem do mundo real". Por consequência, a escolha correta do modelo teórico matemático de uma equação diferencial é capaz de estimar informações futuras e metodizar o comportamento correspondente à variação entre as grandezas estudadas.

Zill (2016) no que diz respeito à dinâmica de populações, utiliza-se por referência o modelo de Malthus, que foi formulado originalmente para modelar o crescimento populacional humano. Este modelo parte da hipótese de que a taxa de crescimento $(r)$ na qual a população $(P)$ de um país cresce em um determinado instante $(t)$ é proporcional à população total do país naquele mesmo instante. Logo, o modelo de Malthus é definido pela seguinte equação diferencial de primeira ordem:

$$
\frac{d P}{d t}=r P
$$

Este modelo conduz a um crescimento exponencial, uma vez que sua solução sujeita à condição inicial $P(0)=P_{o}$ é dada por:

$$
P(t)=P_{o} e^{r t}
$$

Pelo fato de que este modelo não estabelece um limite máximo de indivíduos para a população, afirmando que a população estaria crescendo infinitamente, ele torna-se distante da realidade. Dessa forma, com o passar dos anos, foram surgindo novas adaptações para o modelo Malthusiano, tais como o Modelo Logístico, também conhecido como Modelo de Verhulst (1837).

Conforme nos diz Bassanezi (2009), "O Modelo de Verhulst supõe que uma população, vivendo num determinado meio, deverá crescer até um limite máximo sustentável, isto é, ela tende a se estabilizar". Assim, esse modelo considera que a taxa de crescimento é proporcional à população em cada instante.

Para a análise dos dados, definiu-se de antemão que seria descartado o Modelo de Malthus, pois nas placas utilizadas havia um espaço definido (capacidade de suporte) sendo 
impossível, portanto, que o fungo crescesse para além deste espaço. Portanto, foi utilizado o Modelo de Verhulst, que pode ser expresso por uma equação diferencial com condição inicial, onde $k$ representa a capacidade de suporte:

$$
\left\{\begin{array}{l}
\frac{d P}{d t}=r P\left(1-\frac{P}{k}\right) \\
P(0)=P_{o}, r>0
\end{array} .\right.
$$

A solução analítica da expressão (3) é obtida aplicando-se a integração após a separação das variáveis, assim,

$$
\int \frac{1}{\left(1-\frac{P}{k}\right) \cdot P} d P=\int r d t .
$$

O primeiro termo pode ser resolvido por meio da técnica das frações parciais. Assim é obtida a solução da equação diferencial:

$$
\ln \left|\frac{P}{1-\frac{P}{k}}\right|=r t+C \text {. }
$$

Usando a condição inicial $P(0)=P_{o}$ podemos determinar a constante arbitrária C:

$$
C=\ln \left|\frac{P_{o}}{1-\frac{P_{o}}{k}}\right| .
$$

Logo, substituindo o valor da constante $C$ obtido em (6) na solução da equação diferencial (5), temos que:

$$
\ln \left|\frac{P}{1-\frac{P}{k}}\right|=r t+\ln \left|\frac{P_{o} \cdot k}{k-P_{o}}\right| .
$$

Realizando algumas manipulações algébricas em (7), e explicitando $P(t)$ chega-se na expressão característica do Modelo de Verhulst:

$$
P(t)=\frac{k \cdot P_{o}}{\left(k-P_{o}\right) \cdot e^{-r t}+P_{o}}
$$




\section{Metodologia}

A parte experimental da pesquisa foi realizada no Laboratório de Fitopatologia do IFRS Campus Bento Gonçalves. Nesta primeira fase, foi realizado o preparo de diferentes meios de cultura, a fim de observar o comportamento de Botryosphaeria dothidea.

Com a finalidade de analisar e comparar o crescimento do fungo no decorrer do experimento, utilizou-se como base o meio de cultura BDA (universal para fungos) acrescentandose a ele diferentes componentes com possibilidade de alterar a cinética de crescimento de forma inibitória. É conhecido na literatura o papel antifúngico das nanopartículas (MALLMANN et al., 2015), dessa forma, além da nanopartícula, optou-se por testar outros componentes, a fim de verificar se uma mudança nos componentes do meio de cultura acarretaria também em uma alteração no padrão de crescimento do fungo.

Foram utilizados os seguintes componentes com possibilidade de inibição do crescimento ao meio de cultura BDA: Nitrito de sódio $\left(\mathrm{NaNO}_{2}\right)$, Nitrato de prata $\left(\mathrm{AgNO}_{3}\right)$, Ácido bórico $\left(\mathrm{H}_{3} \mathrm{BO}_{3}\right)$, Borohidreto de sódio não reagente $\left(\mathrm{NaBH}_{4}\right.$ não reagente), Borohidreto de sódio total $\left(\mathrm{NaBH}_{4}\right.$ total) e Nanopartícula de prata 0,05\% (Nano 0,05\%). Para cada componente, incluída a testemunha somente com BDA, fez-se o uso de cinco placas. Utilizando-se dos procedimentos laboratoriais, os diferentes meios de cultura foram vertidos nas placas de Petri, ou seja, pequenas quantidades das concentrações foram transferidas para as placas. Em seguida, com o auxílio de um estilete, o fungo foi repicado nas placas, onde foram feitos pequenos cortes no fungo ativo, catalogado pelo IFRS - Campus Bento Gonçalves, os quais foram depositados no centro das placas, que permaneceram em ambiente laboratorial, sob condições controladas até o final do experimento.

Com os fungos posicionados ao centro das placas, foi possível realizar as medições, impreterivelmente duas vezes ao dia, para a coleta dos dados experimentais, sendo elas feitas em função do seu diâmetro, através do uso de uma régua milimetrada. Alguns dos processos mencionados podem ser observados na Figura 1.

Figura 1 - Experimento.

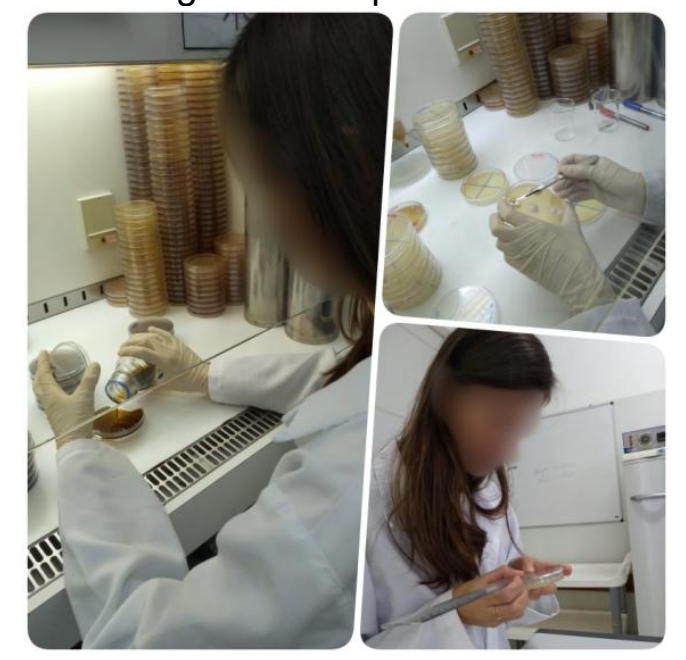

Fonte: Registro fotográfico dos autores (2019). 
As placas foram acompanhadas durante 27 dias e seus dados experimentais foram organizados através de planilhas eletrônicas no software Excel ${ }^{1}$, que possibilitou uma visualização mais detalhada dos dados. Para análises um pouco mais aprofundadas, os dados foram processados no software Scilab², por meio de rotinas computacionais.

\section{Resultados e discussão}

Por meio de recursos computacionais, especialmente com o auxílio dos softwares Excel e Scilab, foi possível a obtenção do gráfico experimental, bem como dos gráficos dos modelos teóricos. Além disso, também calculou-se por meio de uma rotina computacional no Scilab, a taxa de crescimento fúngico $(r)$ e o erro quadrático em relação aos dados experimentais para cada um dos compostos. O cálculo do erro se faz necessário uma vez que para todo e qualquer experimento sempre há um erro a ser levado em consideração, visto que

A acurácia (ou exatidão) e a precisão (número de algarismos significativos do valor medido) de um certo dado medido estarão sempre limitadas tanto pela sofisticação do equipamento utilizado, pela habilidade do sujeito que realiza a medida, pelos princípios físicos básicos tanto do instrumento de medida, quanto do fenômeno que gerou o experimento e o conhecimento que se tem sobre o valor "verdadeiro" da grandeza física. (UNICAMP-FEM, [s.d.], on-line).

No gráfico da Figura 2 pode-se observar o comportamento dos fungos com os diferentes meios de cultura durante o experimento.

Figura 2 - Gráfico experimental das concentrações.

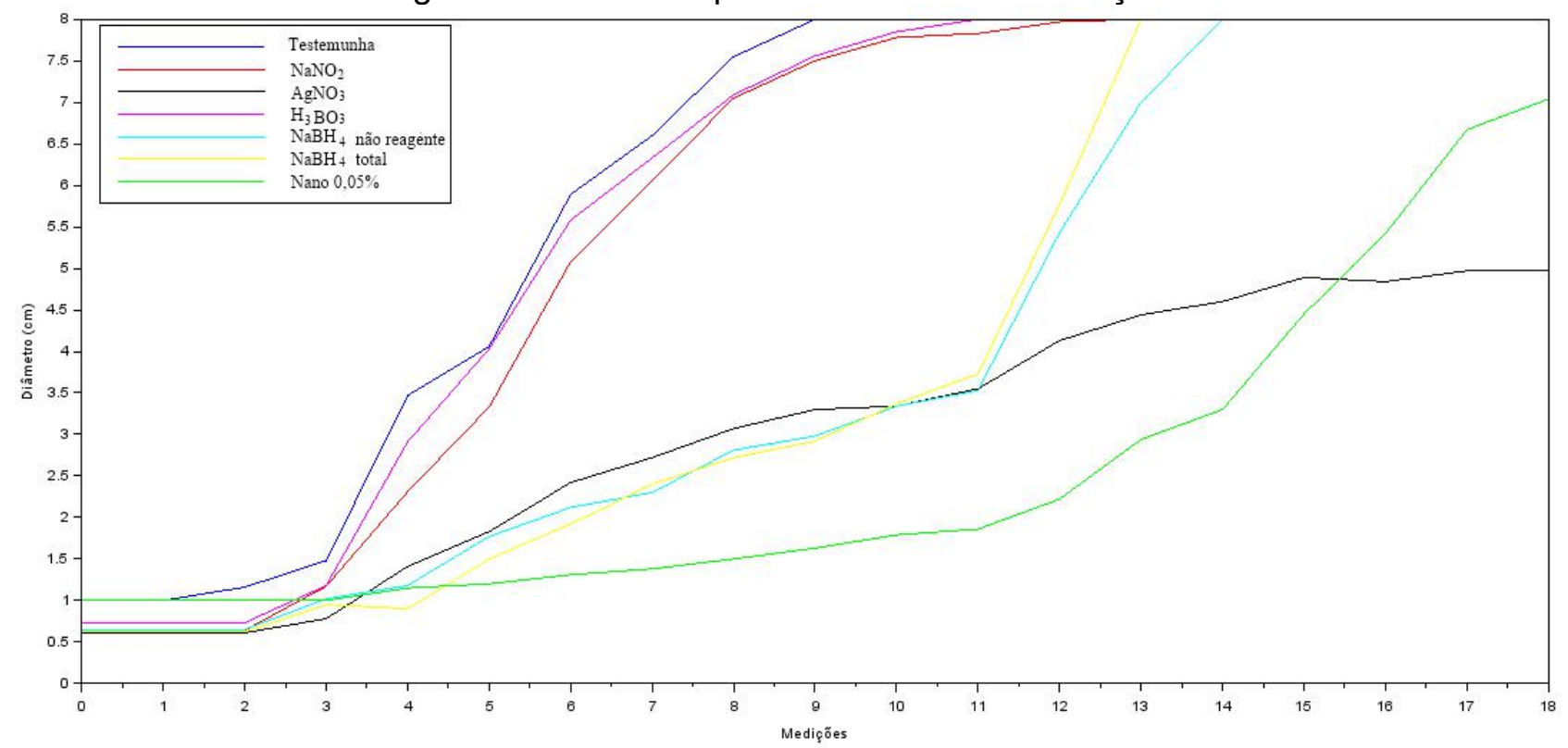

Fonte: Dados da pesquisa (2019).

\footnotetext{
1 Excel é um software desenvolvido pela empresa Microsoft, amplamente usado principalmente por empresas e particulares para a realização de operações financeiras e contábeis usando planilhas eletrônicas. As planilhas são constituídas por células organizadas em linhas e colunas.

2 Scilab é um ambiente voltado para o desenvolvimento de softwares para resolução de problemas numéricos. É um software gratuito e bastante flexível, e está disponível nos mais variados sistemas operacionais. O download do software pode ser feito por meio do site https://www.scilab.org/.
} 
Note que a Figura 2 exibe todos os componentes utilizados no experimento, cada qual apresentando um comportamento diverso em relação à testemunha (fungo mais o BDA), que apresentou maior crescimento em relação às demais. Algumas desenvolveram-se rapidamente, ocupando toda a superfície da placa antes dos 27 dias de experimento, enquanto outras tiveram um retardo no seu crescimento, sequer atingindo a completude. Isto deve se evidenciar quando comparados aos modelos teóricos, que poderão determinar sua taxa de crescimento, bem como o erro quadrático em relação aos dados experimentais.

Em sequência, foram estabelecidas as curvas que representam a evolução do crescimento do fungo, em relação ao tempo, de acordo com o modelo Logístico. Tendo por referência a testemunha (Figura 3), podemos observar que, de acordo com os compostos do meio de cultura, o desenvolvimento do fungo apresenta-se ora mais lento (Figuras 5, 7, 8 e 9) ora mais rápido (Figuras 4 e 6), o que será evidenciado pelas taxas de crescimento obtidas de acordo com o modelo de Verhust. Seguem os gráficos gerados de acordo com o modelo matemático, referente a cada componente utilizado no experimento.

Figura 3 - Curva de crescimento fúngico estimado pelo Modelo de Verhulst em comparação aos dados experimentais obtidos da testemunha.

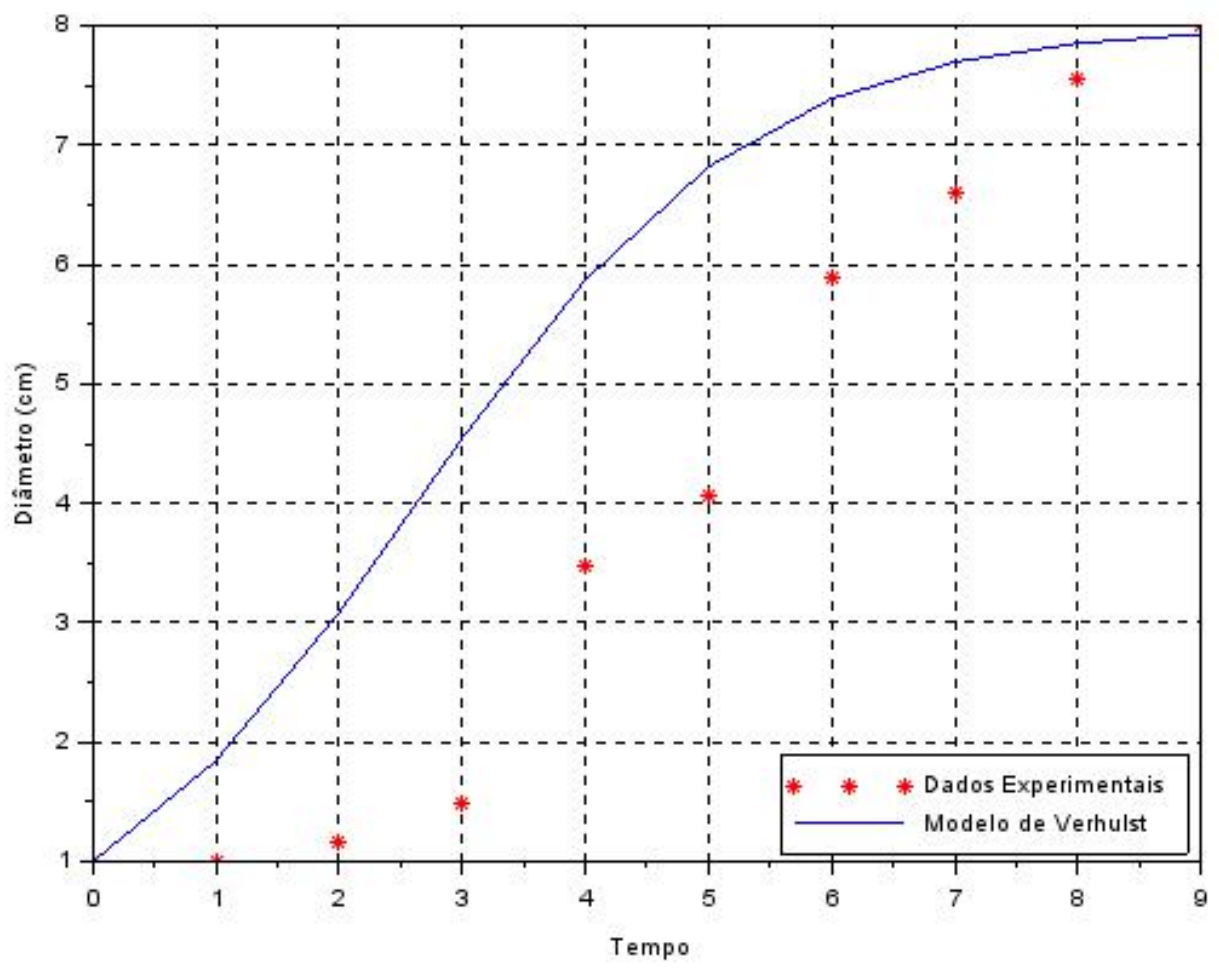

Fonte: Dados da pesquisa (2019). 
Figura 4 - Curva de crescimento fúngico estimado pelo Modelo de Verhulst em comparação aos dados experimentais obtidos da $\mathrm{NaNO}_{2}$.

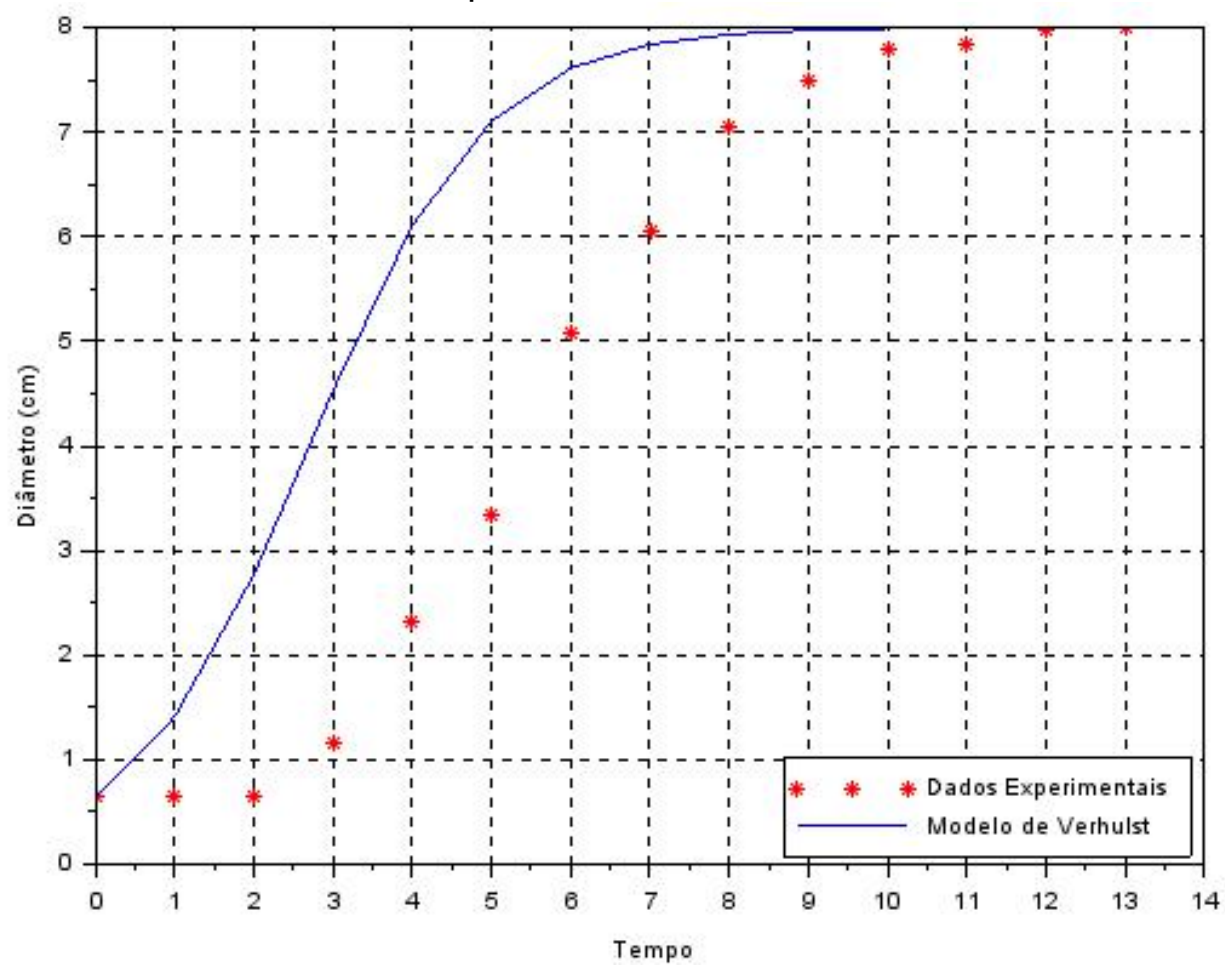

Fonte: Dados da pesquisa (2019).

Figura 5 - Curva de crescimento fúngico estimado pelo Modelo de Verhulst em comparação aos dados experimentais obtidos da $\mathrm{AgNO}_{3}$.

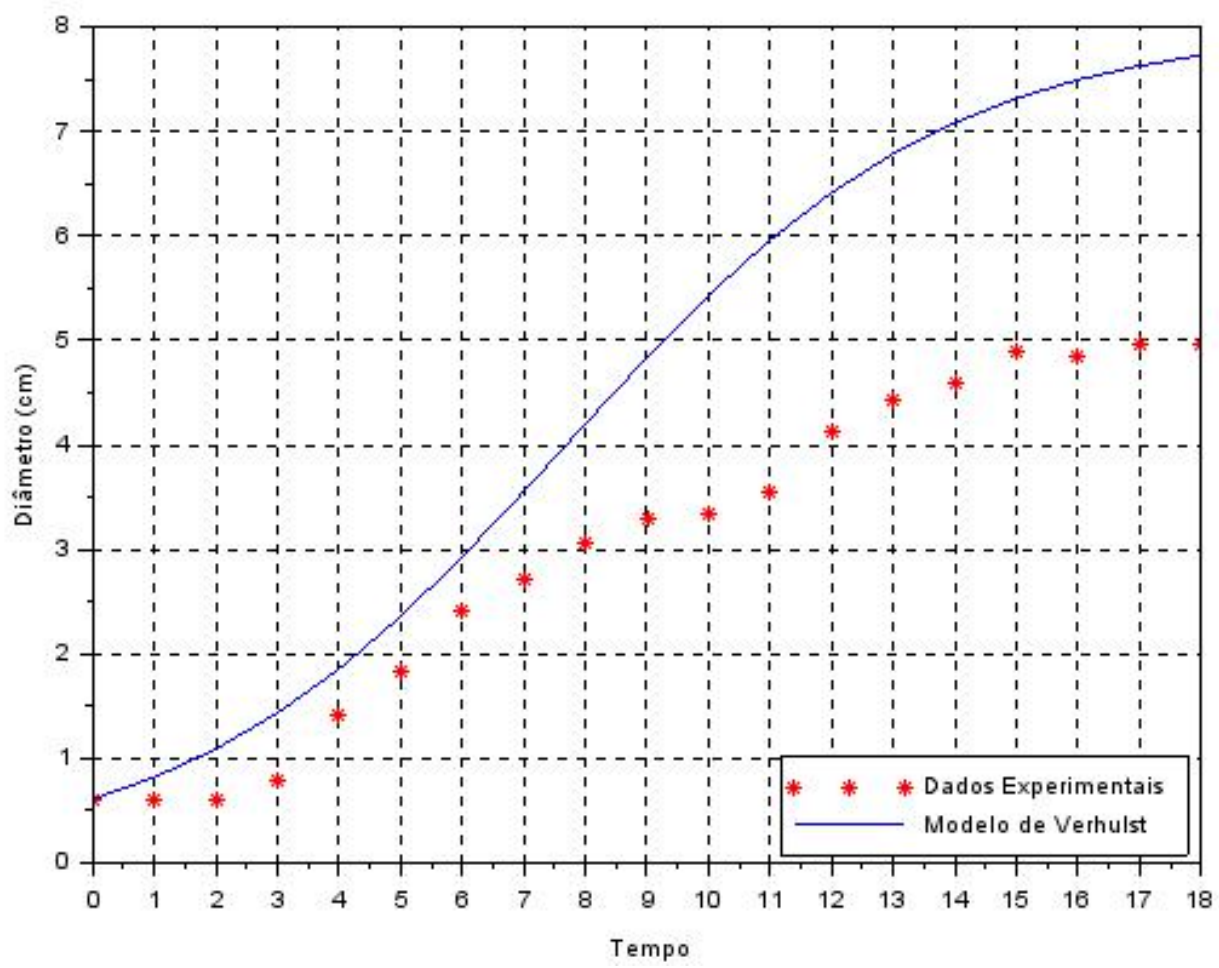

Fonte: Dados da pesquisa (2019). 
Figura 6 - Curva de crescimento fúngico estimado pelo Modelo de Verhulst em comparação aos dados experimentais obtidos da $\mathrm{H}_{3} \mathrm{BO}_{3}$.

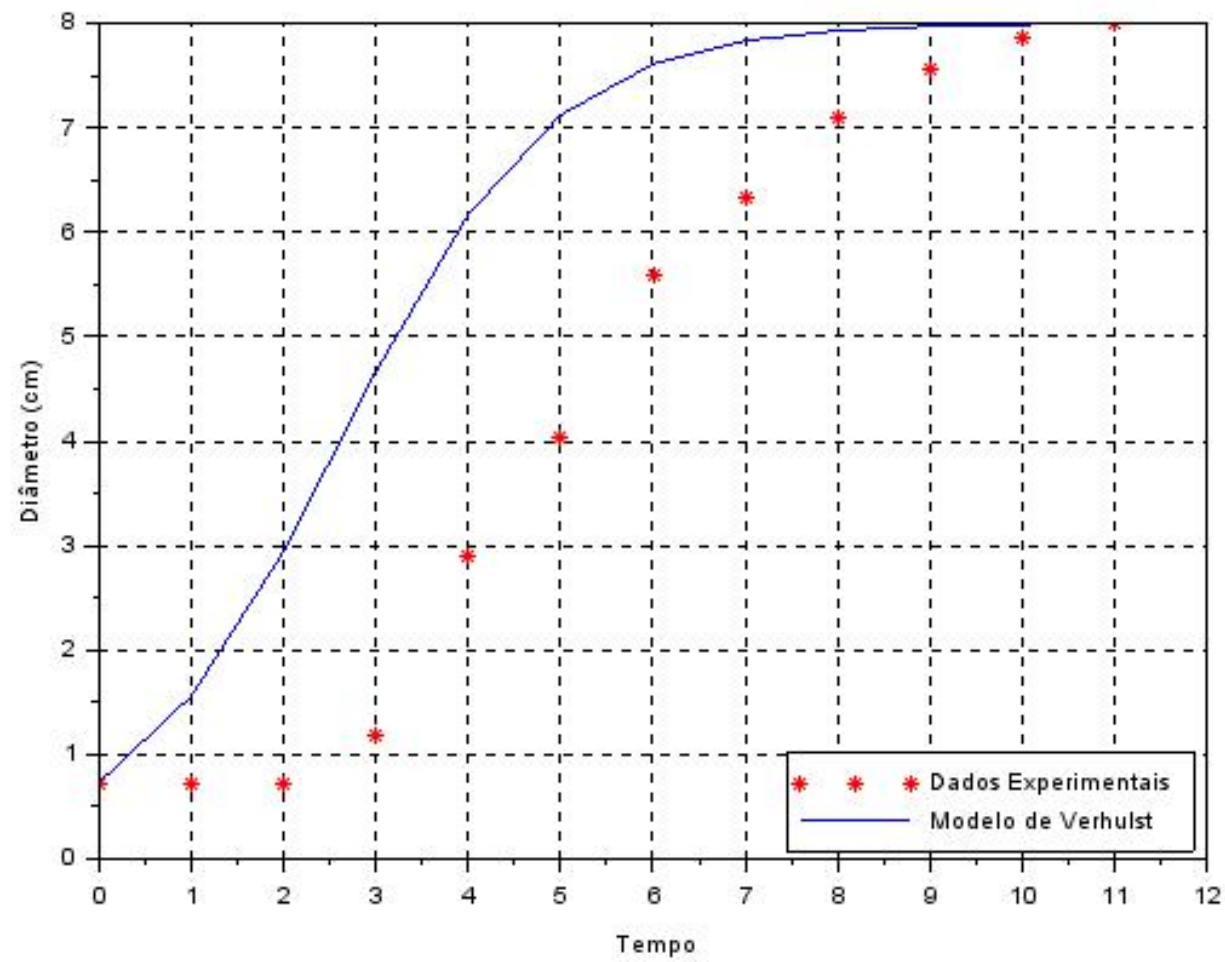

Fonte: Dados da pesquisa (2019).

Figura 7 - Curva de crescimento fúngico estimado pelo Modelo de Verhulst em comparação aos dados experimentais obtidos da $\mathrm{NaBH}_{4}$ não reagente.

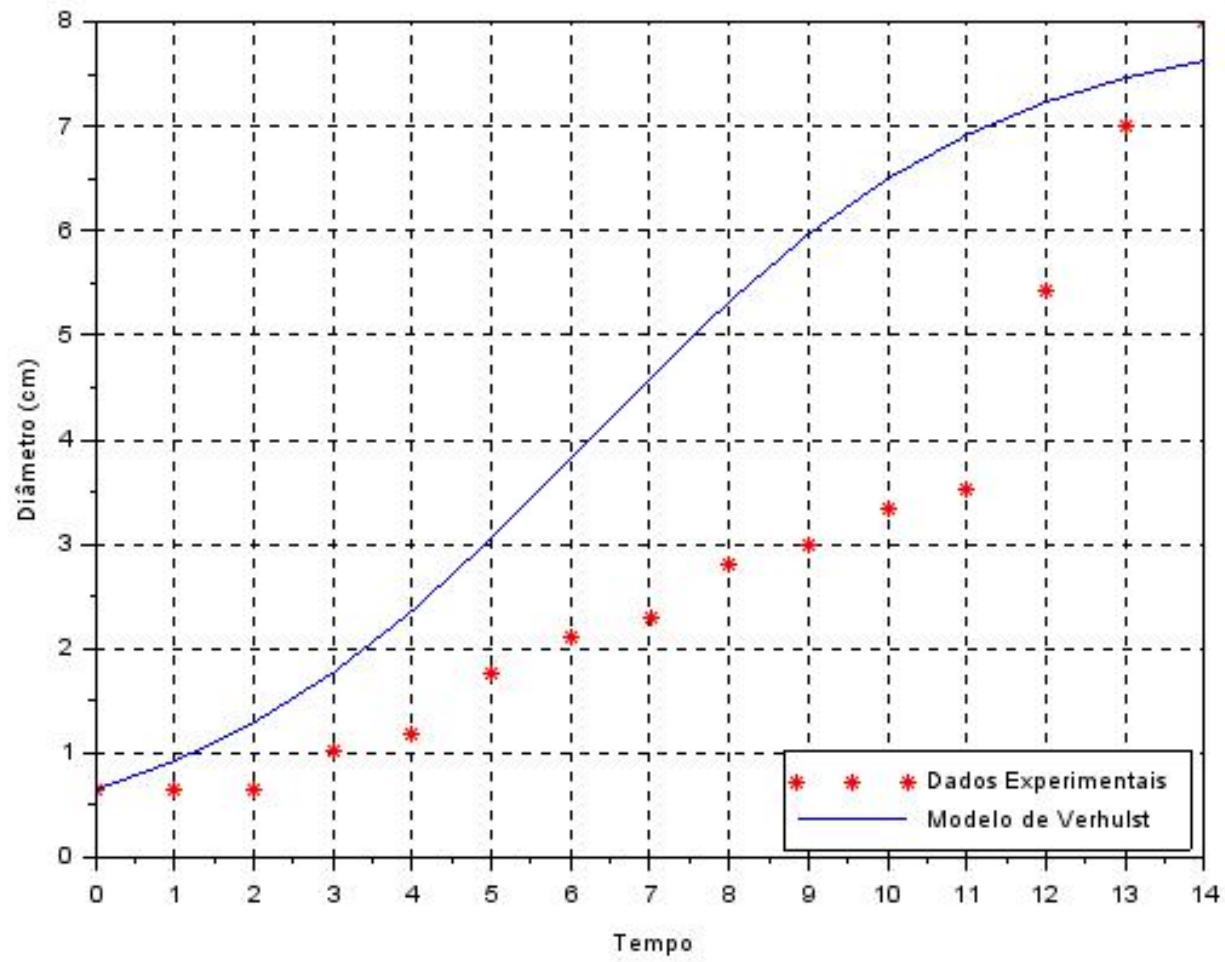

Fonte: Dados da pesquisa (2019). 
Figura 8 - Curva de crescimento fúngico estimado pelo Modelo de Verhulst em comparação aos dados experimentais obtidos da $\mathrm{NaBH}_{4}$ total.

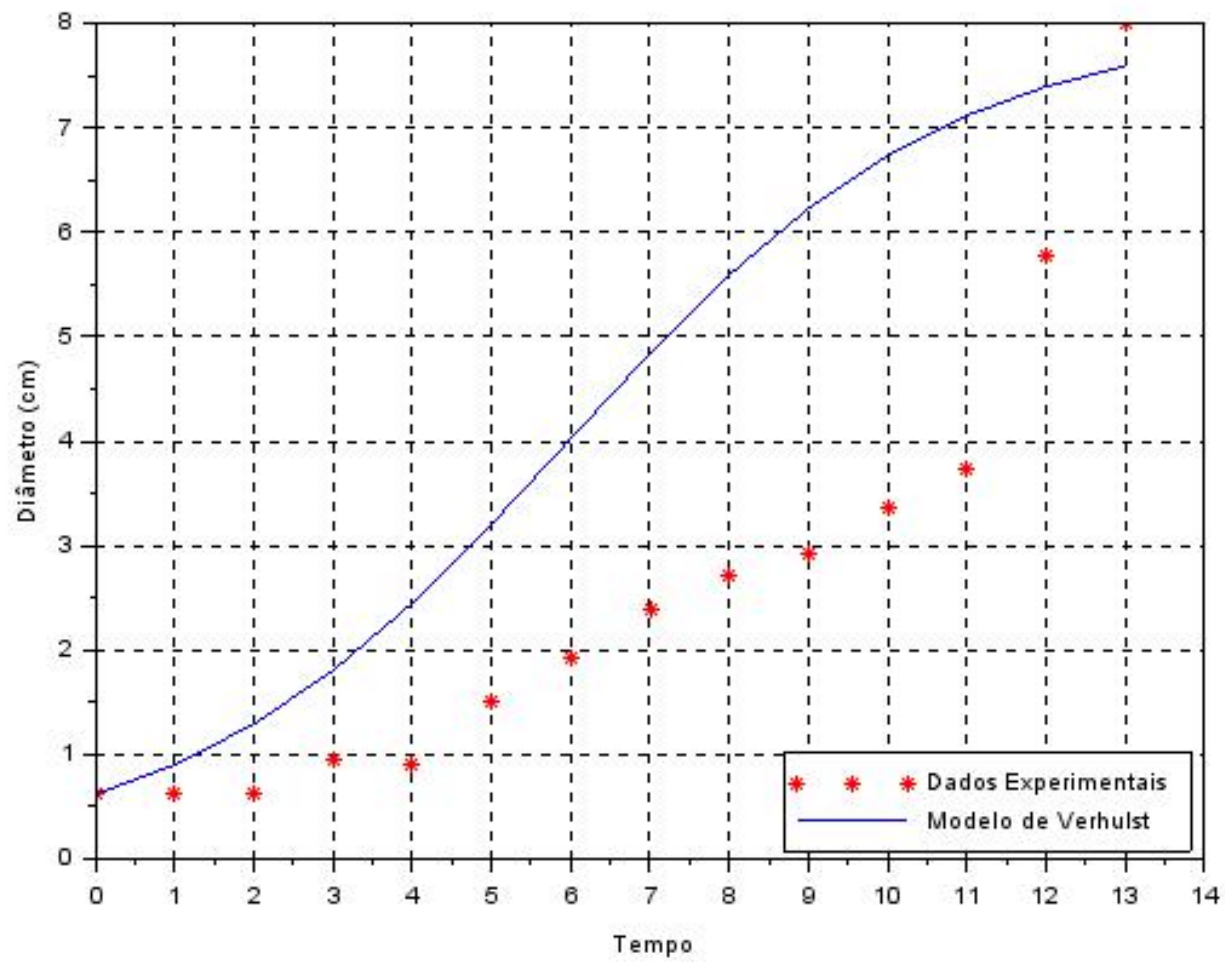

Fonte: Dados da pesquisa (2019).

Figura 9 - Curva de crescimento fúngico estimado pelo Modelo de Verhulst em comparação aos dados experimentais obtidos da Nano $0,05 \%$.

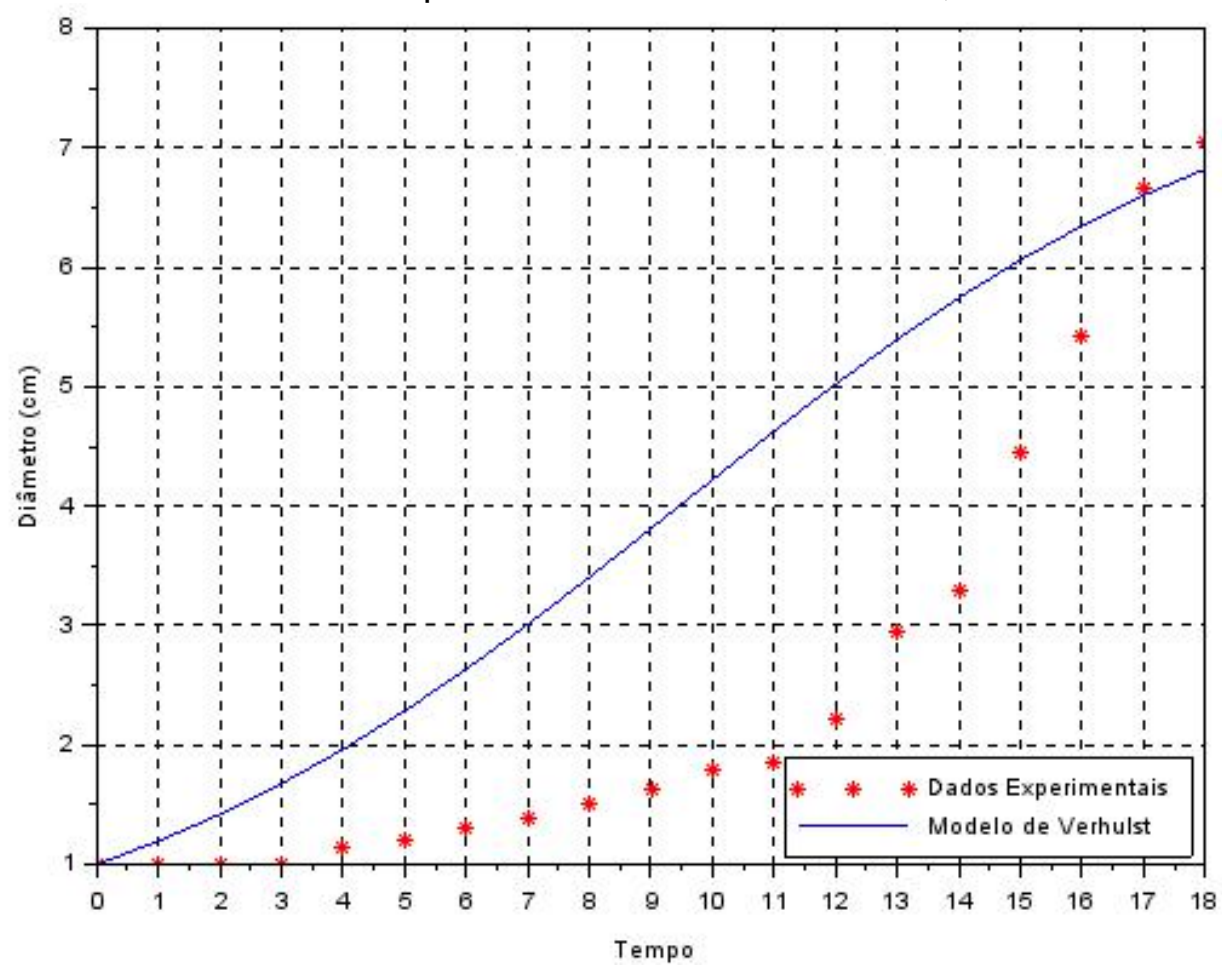

Fonte: Dados da pesquisa (2019).

Após calcular a taxa de crescimento fúngico computacionalmente para cada caso e obter o erro quadrático em relação à curva dada pelo Modelo de Verhulst, organizaram-se esses dados 
em uma tabela comparativa, obtendo, assim, uma visualização mais consistente para alcançar melhores conclusões. Inicialmente, conforme o gráfico experimental das concentrações (Figura 2) acreditava-se que, pelo fato de a testemunha não possuir agente inibidor em sua composição deveria, de acordo com o modelo, ser a responsável pela maior taxa de crescimento em relação aos demais componentes; entretanto, os dados experimentais mostraram-se divergentes do prognóstico esperado. Observe a Tabela 1.

Tabela 1 - Comparação entre as diferentes concentrações.

\begin{tabular}{ccc}
\hline Componentes & $\begin{array}{c}\text { Taxa de } \\
\text { Crescimento }(\boldsymbol{r})\end{array}$ & Erro Quadrático \\
$(\mathbf{c m} / \mathbf{h})$ & 0,73997 & 5,54369 \\
\hline Testemunha & 0,903582 & 7,47041 \\
$\mathrm{NaNO}_{2}$ & 0,324363 & 7,77675 \\
$\mathrm{AgNO}_{3}$ & 0,878332 & 6,72255 \\
$\mathrm{H}_{3} \mathrm{BO}_{3}$ & 0,389006 & 7,24519 \\
$\mathrm{NaBH}_{4}$ não reagente & 0,414823 & 7,85715 \\
$\mathrm{NaBH}_{4}$ Total & 0,205717 & 7,22762 \\
$\mathrm{Nano} 0,05 \%$ &
\end{tabular}

Fonte: Dados da pesquisa (2019).

Como pode-se observar na Tabela 1, o componente que apresentou o crescimento mais lento, de acordo com o modelo de Verhulst foi a Nano $0,05 \%$, pelo efeito da prata como agente antimicrobiano. No entanto, o componente que conteve o crescimento mais acelerado, conforme o modelo, é a $\mathrm{NaNO}_{2}$, superando inclusive a testemunha, o que contradiz o gráfico experimental (Figura 2), não refletindo fielmente a realidade demonstrada. Este crescimento anormal em relação à testemunha poderia ser explicado, dentre outros fatores, pela possibilidade da existência de nutrientes benéficos ao desenvolvimento do fungo na composição do $\mathrm{NaNO}_{2}$. Além disso, a tabela acima nos exibe qual componente obteve o menor erro, sendo ela a testemunha, o que pode ser atribuído ao fato de que o crescimento fúngico se deu de maneira natural, se comparada aos outros compostos com agentes supostamente inibidores.

Com o propósito de fundamentar as informações exibidas na tabela comparativa, observe a Figura 10. Nela está apresentada a comparação de dois dos componentes utilizados no experimento, sendo eles respectivamente a testemunha e a Nano 0,05\%, o componente com crescimento mais lento. 
Figura 10 - Comparação do crescimento do fungo em uma testemunha (esquerda) e em uma placa que contém Nano $0,05 \%$ alocada junto ao meio de cultura (direita).

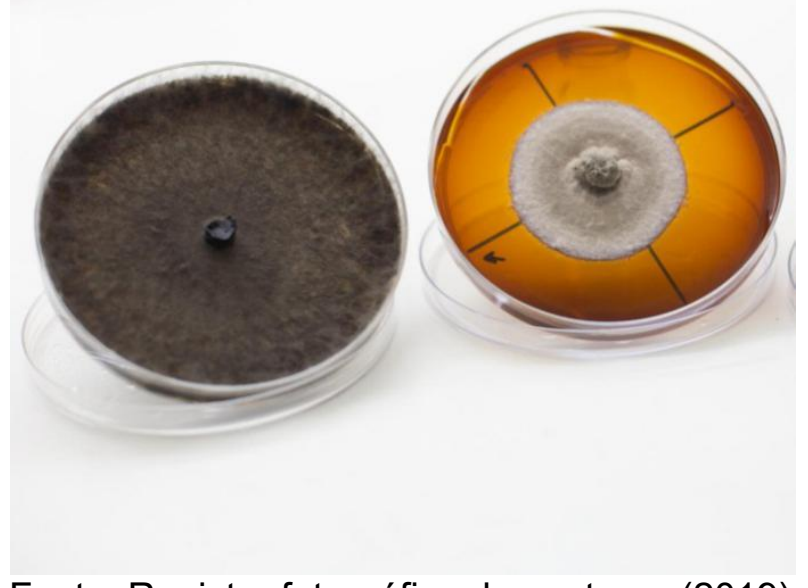

Fonte: Registro fotográfico dos autores (2019).

A primeira placa exibe a testemunha, enquanto a segunda traz a Nano 0,05\%. Pode-se notar que é nítida essa diferença, uma vez que a testemunha apresenta uma taxa de crescimento maior em relação ao composto compreendido pelas nanopartículas.

\section{Considerações finais}

Tendo em vista os aspectos observados e a ideia principal do artigo, que era de conhecer e comparar o comportamento de fungos causadores de doença de tronco em videiras sob a ação de diferentes agentes, foi possível constatar que os meios de cultura que possuíam prata em sua composição ( $\mathrm{Nano} 0,05 \%$ e $\mathrm{AgNO}_{3}$ ) tiveram efeito inibidor no crescimento do fungo, de modo especial a Nano $0,05 \%$.

Observou-se, também, que dentre os componentes agregados ao meio de cultura, a testemunha foi aquela que mais se aproximou do modelo matemático clássico de Verhulst, apresentando o menor erro, no qual acredita-se que possa ser atribuído ao fato dela não possuir agente inibidor em sua composição. Assim, é possível constatar que o fungo Botryosphaeria dothidea aliado ao meio de cultura BDA segue um modelo matemático clássico, uma vez que as curvas geradas mostram seu comportamento, aproximando-se dos dados experimentais. Já o fungo em interação com os demais componentes, que faziam uso de possíveis agentes inibitórios em sua composição, apresentaram um distanciamento maior em relação ao modelo, não retratando fielmente a realidade.

Dessa forma, seriam necessárias adaptações no modelo estudado para obter uma maior precisão acerca do comportamento do fungo na presença dos agentes inibidores. Além disso, 
feitas as adaptações, também seria possível prever com antecedência o comportamento do fungo para o instante de tempo desejado.

Através dos conhecimentos compartilhados no decorrer do processo experimental e de análise, é relevante destacar a importância do trabalho interdisciplinar, uma vez que o trabalho em conjunto possibilita uma rica troca de ideias, proporcionando uma melhor compreensão dos fenômenos, sob os mais variados aspectos. Além disso, essa troca mútua contribui para o desenvolvimento tanto das ciências exatas quanto das ciências agrárias, de modo que novos conhecimentos são gerados, podendo ser utilizados posteriormente. Por fim, este estudo está condicionado a novas pesquisas, a fim de minimizar as discrepâncias entre os dados experimentais e os modelos adotados nas análises, tornando-se necessárias, como citado anteriormente, melhorias e adaptação dos algoritmos computacionais e métodos de medições.

\section{Referências}

ALFENAS, A. C.; MAFIA, R. G. Métodos em Fitopatologia. 2. ed. Viçosa: Editora UFV, 2016.

ALMANÇA, M. A. K.; LERIN, S.; CAVALCANTI, F. R. Doenças da videira. Informe Agropecuário. v. 36, n. 289 , p. $70,2015$.

BASSANEZI, R. C. Ensino-aprendizagem com modelagem matemática. 3. ed. São Paulo: Contexto, 2009.

BOYCE, W. E.; DIPRIMA, R. C. Equações Diferenciais Elementares e Problemas de Valores de Contorno. Tradução de Valéria de Magalhães Lorio. 8. ed. Rio de Janeiro: LTC, 2006.

MALLMANN, E. J. J.; CUNHA, F. A.; CASTRO, B. N. M. F.; MACIEL, A. M.; MENEZES, E. A.; FECHINE, P. B. A. Antifungal Activity of Silver Nanoparticles Obtained by Green Synthesis. Revista do Instituto de Medicina Tropical de São Paulo, São Paulo, v. 57, n. 2, p. 165-167, abr. 2015. DOI: http://dx.doi.org/10.1590/S0036-46652015000200011.

PERIN, R.; FIORENTIN, J.; STROSCHEIN, S.; ALMANCA, M. Equações diferenciais no crescimento de fungos causadores de doenças de tronco em videira. REMAT: Revista Eletrônica da Matemática, Bento Gonçalves, v. 5, n. 2, p. 192-208, 01 jul. 2019. DOI: https://doi.org/10.35819/remat2019v5i2id3376.

UNICAMP. Faculdade de Engenharia Mecânica. Medição de Dados Experimentais, Incerteza e Propagação de Erro: $O$ erro nos dados experimentais. [s.d] Disponível em: http://www.fem.unicamp.br/ instmed/Incerteza.htm. Acesso em: 02 abr. 2020.

ZILL, D. G.; CULLEN, M. R. Equações Diferenciais. Vol. 1. 3. ed. São Paulo: Pearson Makron Books, 2001.

ZILL, D. G. Equações Diferenciais com Aplicações em Modelagem. Vol. 1. Tradução da $10^{a}$ edição norte-americana. Tradução de Márcio Koji Umezawa. 3. ed. São Paulo: Cengage Learning, 2016. 